\title{
INCIDÊNCIA DA SÍNDROME DO COMER NOTURNO E COMPULSÃO ALIMENTAR EM ESTUDANTES DE NUTRIÇÃO
}

\section{Maria Alice Alves de Souza}

Discente da Universidade de Pernambuco (UPE), Campus Petrolina (PE), Brasil.

\section{Verlene Caroline de Souza Gomes}

Discente da Universidade de Pernambuco (UPE), Campus Petrolina (PE), Brasil.

\section{Emerson Iago Garcia e Silva}

Graduado em nutrição pela Universidade de Pernambuco (UPE), Campus Petrolina, Brasil.

Cristhiane Maria Bazílio de Omena Messias

Docente Adjunta do Colegiado de Nutrição e do Programa de Pós-graduação em Formação de Professores e Práticas Interdisciplinares (PPGFPPI) da Universidade de Pernambuco (UPE), Petrolina, (PE), Brasil.

E-mail: cristhiane.omena@upe.br
RESUMO: O presente trabalho objetiva caracterizar a incidência da síndrome do comer noturno e a compulsão alimentar em estudantes do curso de nutrição. Trata-se de um estudo transversal de caráter analítico realizado em uma instituição de nível superior em Petrolina -PE. Foram aplicados três questionários: questionário de hábitos do comer noturno, a escala de ingestão compulsiva e o The Three factor eating questionnaire. Avaliaram-se 81 estudantes de ambos os sexos, com predominância do sexo feminino. Não houve associação significativa entre a síndrome do comer noturno ou a compulsão alimentar com as variáveis sexo e escolaridade. Em relação às variáveis comportamentais, a alimentação emocional foi o fator melhor relacionado ao descontrole alimentar e a restrição cognitiva tem pouca contribuição para o agravamento da alimentação descontrolada. $\mathrm{O}$ investimento no estudo dos transtornos alimentares é importante para a criação de uma intervenção adequada, com o intuito de reduzir incidência tanto da SCN como da compulsão alimentar.

PALAVRAS-CHAVE: Transtorno da compulsão alimentar; Estudantes; Transtornos da Alimentação e da Ingestão de Alimentos.

\section{OCCURRENCE OF NIGHT-EATING SYNDROME AND FEED COMPULSIVE DISORDER IN NUTRITION STUDENTS}

\begin{abstract}
Occurrence of night-eating syndrome and feed compulsive disorder in students of the Nutrition Course is analyzed in current transversal and analytic study at a higher education institute in Petrolina PE Brazil. Three questionnaires were applied, namely, a questionnaires on habits in night-eating, compulsive ingestion scale and the Threefactor eating questionnaire. Eighty-one students, males and females, mostly the latter, were evaluated. There was no significant association between night-eating syndrome or feed compulsive disorder for the variables gender and schooling. In the case of behavioral variables, emotional feeding was the factor which was most related to lack of control in feeding, whilst cognitive restriction scantily contributed towards the worsening of uncontrolled feeding. Studies on feeding disorders are important for an adequate intervention to reduce occurrence in NES and feeding compulsive disorder.
\end{abstract}

KEY WORDS: Binge-Eating Disorder; Students; Feeding and Eating Disorders. 


\section{INTRODUÇão}

$\mathrm{O}$ aumento da prevalência dos transtornos alimentares (TA) tem sido ressaltado nos últimos anos, em que houve constatação do aumento na frequência de problemas associados à imagem corporal e comportamentos alimentares anormais entre os adolescentes (COSTA et al., 2009). Os transtornos alimentares possuem uma etiologia multifatorial, composta de predisposições genéticas, socioculturais e vulnerabilidades biológicas e sociológicas. Geralmente, apresentam as suas primeiras manifestações na infância e na adolescência e ocorrem com muita frequência entre os jovens universitários (ZIROLDO et al., 2011).

Entre os diversos transtornos alimentares destacamos a Síndrome do Comer Noturno (SCN) que, segundo Milano et al (2012), é caracterizada por uma alimentação reduzida durante o dia, à noite hiperfagia acompanhada por despertares noturnos frequentes, associados com episódios conscientes de compulsiva ingestão de alimentos, ritmos circadianos anormais e outros fatores neuroendócrinos.

A prevalência de SCN é estimado em pouco mais de $1 \%$ na população em geral, entre 6 e $14 \%$ em pacientes obesos, caracterizando humor deprimido, duas ou mais complicações de diabetes e também de baixa adesão a dieta. Além disso, como mostram também se incorporam como características prevalentes o fato de ocorrer mais em mulheres, aparecendo na infância ou na adolescência (CALUGI et al., 2013; NOLAN; GELIEBTER, 2012).

Segundo Cleator et al. (2012), a SCN pode se associar com estresse e ansiedade, modulando uma série de comportamentos que atestam a necessidade de intervenções terapêuticas, buscando reconhecer a gravidade deste distúrbio, para proporcionar sempre o bem-estar social, físico e mental dos pacientes.

O tratamento da SCN é bem complexo e por isso deve ser orientado em base individual, combinando a terapia de saúde mental, educação nutricional, e com o possível uso de fármacos para reduzir o estresse, além de exames laboratoriais para análise do sono, para então possibilitar diagnósticos mais precisos (HARB et al., 2010).

Estudar esses transtornos alimentares clinicamente significativos, como a SCN, permite a avaliação e o planejamento do tratamento de determinadas patologias associadas de forma mais racional e individualizada. Os desequilíbrios circadianos reforçam a ligação entre a SCN com a obesidade, assim, teoricamente a SCN poderia levar a ganho de peso sendo resultado do excesso de calorias consumidas durante a noite. Com efeito, estudos têm mostrado que o comer noturno leva ao ganho de peso e que o início da obesidade ocorre mais cedo na vida daqueles com a síndrome (GALLANT et al., 2012).

Porém a psicopatologia da $\mathrm{SCN}$ não é apenas pelo sobrepeso ou obesidade, uma vez que também está presente em indivíduos com peso normal que possuem elevados níveis de estresse crônico, levando-os a evitar noites angustiantes com a ingestão de alimentos que geram conforto por possuir altos valores calóricos pelos seus hidratos de carbono e teor de gordura (MILANO et al., 2012).

Ainda no contexto de padrões alimentares irregulares, é importante ressaltar sobre os indivíduos com compulsão alimentar periódica (TCAP), que em alguns estudos vêm sendo relacionado com sobrepeso e obesidade, exercendo importante papel na saúde e peso corporal. A compulsão alimentar periódica é caracterizada pelo consumo de uma grande quantidade de alimentos em até 2 horas, seguida pela sensação de perda do controle, tristeza e culpa após comer e ausência de comportamentos compensatórios. Inclui anormalidades fisiológicas na ação de hormônios que influenciam a ingestão alimentar, como insulina, leptina, grelina entre outros. Tem sido observado na prática clínica que os pacientes obesos relatam o estresse como um fator para o início da compulsão alimentar e outras desordens alimentares (BERNARDI et al., 2009).

Em resposta à regulação do estresse, os peptídeos como a leptina e a grelina, entre outros, estão envolvidos de forma direta na ingestão alimentar e a interação dos dois tem sido estudada em humanos. Estudos avaliaram obesos e não obesos com TCAP e sem TCAP indicando que o estresse pode induzir aumento nos níveis de grelina plasmática, associado a níveis séricos de cortisol e levando os indivíduos a consumir mais alimentos em menores intervalos de tempo (BERNARDI et al., 2009).

Segundo Colles et al. (2007), estas síndromes têm sido positivamente correlacionadas com um padrão de refeição irregular, caracterizado por um atraso do horário das refeições devido ao stress. O que sugere que 
as exigências do dia a dia, associadas a maiores níveis de ansiedade e estresse próprios das tarefas desempenhadas em níveis de escolaridade superior, induzem à alteração do horário das refeições. O deslocamento por parte dos sujeitos referente ao consumo alimentar diário para o período da noite, facilita o envolvimento em comportamentos de hiperfagia e ingestão compulsiva.

A adoção de condutas e o estilo de vida diferenciado podem tornar os universitários grandes consumidores da alimentação fora do domicílio e possivelmente um grupo vulnerável às circunstâncias que coloca em risco sua saúde e qualidade de vida. (DUARTE et al., 2013). É sabido que padrões comportamentais e emocionais disfuncionais tendem a surgir com muita frequência entre os jovens, talvez como uma "válvula de escape" para frustrações no desempenho escolar ou como compensação pelas exigências dos pais e da sociedade (MARQUES, 2013).

Esse aumento de prevalência dos transtornos alimentares, principalmente entre a população estudantil, tem levado inúmeros pesquisadores a intensificar seus estudos nesse campo de investigação em busca de um conhecimento mais aprofundado sobre as causas, a evolução, o tratamento, as possibilidades de recuperação desses quadros, bem como sobre as consequências do TA para a vida social e educacional das pessoas acometidas por eles (COSTA et al., 2009).

Neste contexto, o presente estudo teve como objetivo caracterizar a incidência da síndrome do comer noturno e a compulsão alimentar em estudantes de nutrição de uma instituição pública de ensino superior.

\section{METODOLOGIA}

Trata-se de um estudo transversal de caráter analítico desenvolvido no período de agosto de 2015 a julho de 2016, em uma instituição pública de ensino superior. A amostra foi constituída por 81 estudantes universitários do curso de nutrição, sendo 67 do sexo feminino e 14 do sexo masculino. Foram excluídos do presente estudo aqueles que não se apresentaram devidamente matriculados.

Este subprojeto pertence a um projeto intitulado "Incidência da síndrome do comer noturno e compulsão alimentar em estudantes da área de saúde e funcionários de uma instituição pública de ensino superior do município de Petrolina - PE", aprovado pelo Comitê de Ética da Universidade de Pernambuco, CAAE: 43757215.1.0000.5207; em junho de 2015.

Inicialmente, a proposta do projeto foi apresentada aos participantes e as dúvidas esclarecidas. Em seguida os alunos receberam o Termo de Consentimento Livre e Esclarecido (TCLE). Após o retorno do TCLE devidamente assinado iniciou -se a coleta de dados. Para a coleta, foram aplicados três questionários: questionário de hábitos do comer noturno (QHCN), (HARB et al., 2008), escala de ingestão compulsiva (EIC) traduzida por Freitas et al. (2001) e The Three factor eating questionnaire (TFEQ R-21) (NATTACCI; JÚNIOR, 2011). A aplicação ocorreu em dias distintos e conforme a disponibilidade dos alunos. O preenchimento durou em média de 20 a 30 minutos.

O questionário de hábitos do comer noturno é um instrumento de avaliação do comportamento alimentar de indivíduos no período da noite. A versão brasileira, adaptada e traduzida por Harb et al (2008), apresentou consistência satisfatória, atestando a fiabilidade do instrumento. É composto por 14 itens, em escala do tipo Likert com cinco opções de resposta (04). O score final é calculado revertendo os valores das questões $1,4 \mathrm{e}$ 14, podendo variar entre 0 a 56 . À medida que o valor total é maior, mais provável está o paciente a apresentar a Síndrome do Comer Noturno (SCN). Um score de 25 ou mais sugere a existência da SCN e um score igual ou superior a 30 é um fator indicador de SCN.

A Escala de Ingestão Compulsiva (BES) é um questionário de auto relato amplamente utilizado para avaliar comportamento e as características da Perturbação de Ingestão Compulsiva (PIC) em pacientes obesos (FREITAS et al.,2001). Esse instrumento tem sido utilizado em vários contextos (MARQUES,2013). A EIC, inicialmente, foi desenvolvida em países de língua inglesa e, posteriormente, adaptada e traduzida para a população brasileira por Freitas et al (2001), sendo validada mais recentemente numa amostra de obesos por Tapadinhas e Pais Ribeiro (2008).

Refere-se a uma escala do tipo Likert com 16 itens, sendo dois deles constituídos por três afirmações 
e o restante de quatro. Cada afirmação pode ser cotada desde 0 (ausência) até 3 pontos (gravidade máxima), sendo que nos itens com apenas três afirmações a cotação seria de 0 a 2. Para cada item deve ser selecionada a afirmação que mais corresponde aos hábitos alimentares do indivíduo (MARQUES, 2013).

A pontuação final é o resultado da soma dos 16 itens, podendo variar entre 0 e 46 pontos, permitindo assim a avaliação dos indivíduos de acordo com os scores pré-definidos. São classificados como ausência de PIC uma pontuação inferior ou igual a 17; PIC moderada uma pontuação entre 18 e 26; e PIC grave uma pontuação maior ou igual a 27 (FREITAS et al., 2001; TAPADINHAS; RIBEIRO, 2008; MARQUES,2013).

O The Three Factor Eating Questionnaire (TFEQ R-21) - questionário de três fatores alimentares vem sendo proposto como um meio eficaz para avaliar estes três padrões de comportamento associados ao hábito alimentar, restrição cognitiva (RC- restrição intencional do consumo alimentar para regulação ponderal), alimentação emocional (AE - consumo alimentar desencadeado por sentimentos) e o descontrole alimentar (DA - perda de critério em relação à seleção, frequência, qualidade e quantidade de alimentos consumidos) (NATTACCI, 2009).

Os questionamentos utilizados possibilitaram a compreensão do comportamento alimentar noturno, os padrões de ingesta calórica durante o dia, as calorias ingeridas após o jantar, sentimentos depressivos e alterações do sono, todos esses aspectos foram importantes na análise dos indivíduos que tendem apresentar ou apresentam algum transtorno alimentar (MARQUES, 2013).

Posteriormente, os dados coletados foram tabelados no Programa Microsoft Excel 2010. A análise estatística foi realizada através do Programa BioEstat 5.0, que se analisaram as variáveis dependentes: Questionário de Hábitos do Comer Noturno, Escala de Ingestão Compulsiva, The Three Factor Eating Questionnaire R-21 e as variáveis independentes de gênero e escolaridade. Para avaliar a associação entre o sexo e as variáveis categorizadas: Questionário de Hábitos do Comer Noturno e Escala de Ingestão Compulsiva, aplicouse o teste exato de Fisher, já que o somatório entre as frequências esperadas e observadas foi $<30$. Para averiguar as associações entre os scores das variáveis comportamentais, restrição cognitiva (RC), alimentação emocional (AE) e descontrole alimentar (DA) obtida pela análise The Three Factor Eating Questionnaire R-21 foi utilizado o teste de regressão linear e coeficiente de correlação de Pearson. Consideraram-se significativos os valores que apresentaram $p<0,05$.

Ainda, foi utilizado o coeficiente alfa de Cronbach (1951), desenvolvido para calcular a confiabilidade de um teste em situações em que o pesquisador não tem a oportunidade de fazer outra entrevista com o indivíduo, validando os resultados obtidos, dando maior robustez ao estudo, tornando o instrumento mais confiável para utilização em estudos científicos (ALMEIDA; SANTOS; COSTA, 2010).

\section{RESULTADOS}

Nesse estudo realizou-se uma análise da presença da síndrome do comer noturno e compulsão alimentar em universitários do curso de nutrição.

Nos resultados apresentados a diferença entre as variáveis de sexo e período de escolaridade não foi estatisticamente significante, ao todo, deve-se ter em mente que as dimensões da população estudada foram relativamente pequenas, principalmente quando consideramos a amostra de 67 mulheres (83\%) para apenas 14 do sexo masculino (17\%). Portanto, diferenças significativas não foram detectadas.

A síndrome está vinculada ao controle da fome e da saciedade e à dessincronização entre o ritmo alimentar e o ritmo sono/vigília, a compreensão do seu processo gênico poderá demonstrar o impacto na alimentação e no processo saúde-doença, incorporam-se como características prevalentes da SCN o fato de ocorrer mais no sexo feminino; na amostra, o sexo feminino teve 6\% de presença/risco de SCN e o sexo masculino teve $0 \%$ de presença/risco de SCN, em que a população de estudo teve $P>0,05$ não indicando relação com essa variável (Tabela 1). 
Tabela 1. Distribuição da síndrome do comer noturno por sexo e escolaridade em universitários, Petrolina - Pernambuco, 2016

\begin{tabular}{ccccc}
\hline & $\begin{array}{c}\text { Presença } \\
\text { ou risco }\end{array}$ & Ausência & Total & $p$-valor* \\
\hline \multicolumn{5}{c}{ Sexo } \\
\hline Feminino & 4 & 63 & 67 & $p=0,5969$ \\
Masculino & 0 & 14 & 14 & \\
\hline \multicolumn{5}{c}{ Escolaridade (Períodos) } \\
\hline $1^{\circ}$ e $3^{\circ}$ & 1 & 33 & 34 & \\
$5^{\circ}$ e $7^{\circ}$ & 3 & 44 & 34 & 0,6355 \\
\hline $\begin{array}{c}\text { Total } \\
(\text { Geral) }\end{array}$ & 4 & 77 & 81 & \\
\hline
\end{tabular}

* Teste exato de Fischer.

Fonte: Dados da pesquisa

A população estudantil possui características e vulnerabilidades que podem desencadear a SCN; maiores níveis de escolaridade impõem ritmos e estilos de vida diferenciados, com frequente presença de estresse e desregularidade dos horários das refeições podendo ocorrer a síndrome. A população estudada foi dividida entre os períodos iniciais $\left(1^{\circ}\right.$ e $\left.3^{\circ}\right)$ com $3 \%$ de presença/ risco e os períodos finais $\left(5^{\circ}\right.$ e $\left.7^{\circ}\right)$ com $7 \%$ de presença/ risco, logo os resultados não são estatisticamente significantes em relação à presença da síndrome (Tabela $1)$.

A compulsão alimentar periódica (CAP) foi uma ferramenta utilizada para avaliar a perda de controle alimentar bem como episódios de compulsão nos estudantes, em que o percentual encontrado para compulsão alimentar na forma moderada/grave foi de $3 \%$ no sexo feminino e $7 \%$ no sexo masculino, quando relacionamos com a variável escolaridade, os períodos iniciais $\left(1^{\circ}\right.$ e $\left.3^{\circ}\right)$ tiveram $3 \%$ da presença moderada/grave da CAP e $7 \%$ em períodos finais $\left(5^{\circ}\right.$ e $\left.7^{\circ}\right)$. Portanto, as variáveis sexo e escolaridade não apresentaram correlação à compulsão alimentar (Tabela 2).

Tabela 2. Distribuição da presença/risco da compulsão alimentar periódica por sexo e escolaridade em universitários, Petrolina - Pernambuco, 2016

\begin{tabular}{|c|c|c|c|c|}
\hline & Ausência & Moderada/Grave & Total & $p$-valor* \\
\hline \multicolumn{5}{|c|}{ Sexo } \\
\hline Feminino & 65 & 2 & 67 & \multirow{2}{*}{$p=0,4343$} \\
\hline Masculino & 13 & 1 & 14 & \\
\hline \multicolumn{5}{|c|}{ Escolaridade (Períodos) } \\
\hline $1^{\mathrm{o}}$ e $3^{\mathrm{o}}$ & 33 & 1 & 34 & \multirow{2}{*}{$p=1.0000$} \\
\hline $5^{\circ}$ e $7^{\circ}$ & 45 & 2 & 47 & \\
\hline Total (Geral) & 78 & 3 & 81 & \\
\hline
\end{tabular}

* Teste exato de Fischer.

Fonte: Dados da pesquisa

A relação dos valores encontrados da CAP, de acordo com o período em que a população se encontrava, teve valor de $P$ maior que os níveis 0,05 na análise estatística; os dados são consistentes com a hipótese nula, não existindo evidência para indicar que o período da escolaridade afeta na compulsão alimentar periódica.

$\mathrm{Na}$ identificação dos comportamentos alimentares $\mathrm{AE}, \mathrm{DA}$ e $\mathrm{RC}$ e a associação entre essas variáveis comportamentais, o estudo mostrou resultados poucos expressivos quanto à significância da relação restrição cognitiva, alimentação emocional e descontrole alimentar; já a relação entre a alimentação emocional e o descontrole alimentar teve o valor de $P<0,05$, indicando resultado significativo entre estas variáveis (Tabela 3). 
Tabela 3. Regressão linear e coeficiente de correlação de Pearson das variáveis comportamentais de estudantes universitários, Petrolina - Pernambuco, 2016

\begin{tabular}{cccc}
\hline Componentes & $\begin{array}{c}\text { Cor. linear de Pearson } \\
(r \text {-valor })\end{array}$ & $p$ - valor (correlação linear) & $\begin{array}{c}p \text { - valor regressão linear } \\
(p \text {-valor })\end{array}$ \\
\hline AE e DA & 0,4473 & $P<0,0001$ & $P=0,0001$ \\
RC e AE & $-0,0136$ & $P=0,9056$ & $P=0,914$ \\
RC e DA & 0,0190 & $P=0,8683$ & $P=0,8627$ \\
\hline
\end{tabular}

Na Figura 1, as imagens A, B e C expressam os diagramas de dispersão para cada par de variáveis comportamentais com a respectiva reta de ajuste dos modelos de regressão linear e o coeficiente de correlação linear de Pearson específico. A avaliação da associação dos comportamentos entre si aponta correlação mais forte entre AE e DA. Não foram encontradas correlações estaticamente significantes entre o comportamento de $\mathrm{RC}$ com AE e/ou DA.
(A)

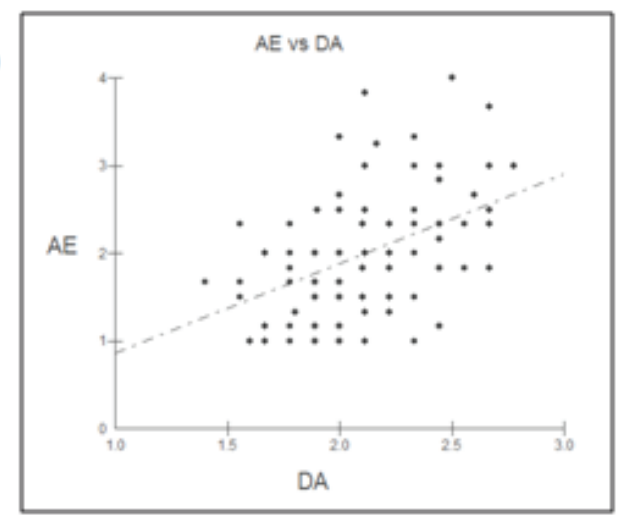

(C)

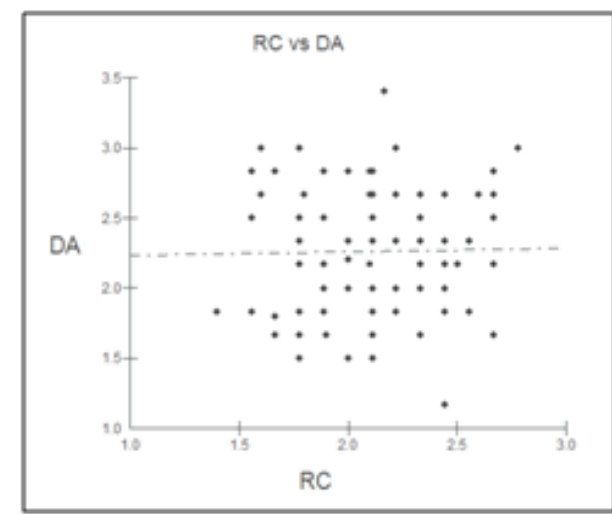

(B)

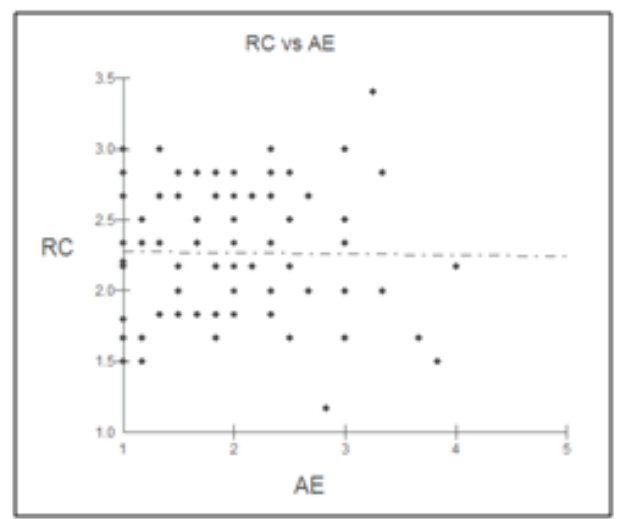

Figura 1. Nível de dispersão, regressão linear e índices de correlação de Pearson entre pares dos domínios comportamentais do hábito alimentar - (A) Alimentação emocional (AE) e Descontrole alimentar (DA); (B) Restrição cognitiva (RC) e Alimentação emocional (AE) e (C) Restrição cognitiva (RC) e Descontrole alimentar (DA) de estudantes de nutrição, Petrolina, 2016.

Ao avaliar a consistência interna dos questionários (Quadro 1), o coeficiente $\alpha$ é calculado a partir da variância dos itens individuais e da variância da soma dos itens de cada avaliador de todos os itens de um questionário que utilizem a mesma escala de medição, desta forma quanto mais o coeficiente alpha se aproximar da unidade mais consistente é o instrumento. No estudo, os resultados indicaram uma boa adequação do instrumento traduzido para os seus propósitos. 
Quadro 1. Valores do coeficiente de alpha de Cronbach em relação aos instrumentos utilizados, Petrolina - Pernambuco, 2016

\begin{tabular}{|c|c|c|}
\hline Instrumento & $\begin{array}{c}\text { Alfa de } \\
\text { Cronbacb }\end{array}$ & $\begin{array}{c}\text { Confiabilidade } \\
\text { interna }\end{array}$ \\
\hline $\begin{array}{c}\text { Questionário de hábitos } \\
\text { do comer noturno }\end{array}$ & 0,5889 & Baixa \\
\hline $\begin{array}{c}\text { The Three factor eating } \\
\text { questionnaire }\end{array}$ & 0,6871 & Moderada \\
\hline $\begin{array}{c}\text { Escala de ingestão } \\
\text { compulsiva }\end{array}$ & 0,7916 & Alta \\
\hline
\end{tabular}

\section{DISCUSSÃO}

A síndrome do comer noturno e a compulsão alimentar, relacionada aos estudantes de nutrição, foram a base do estudo, cujos grupos estudados e relacionados (sexo, escolaridade e período) não apresentaram associações estatisticamente significantes, o que pode ser pela pequena amostra ou decorrente de um efeito de confusão com os comportamentos alimentares.

Estudos têm apontado para maior incidência de padrões de comer noturno na população feminina. Podese levantar essa hipótese, uma vez que as oscilações dos hormônios sexuais, contribuem para comportamentos alimentares irregulares. Harb et al. (2010) confirmam esta tendência, afirmando que $60 \%$ dos pacientes com síndrome do comer noturno são mulheres.

Segundo Marques (2013), outro ponto de relação com a SNC refere-se aos níveis de escolaridade, demonstrando que sujeitos com maior grau de escolaridade são menos propensos a desenvolver a síndrome, tendo em vista que os indivíduos com elevada instrução parecem fazer melhor uso da informação associada à saúde, no sentido em que a educação fornece maior acesso à informação e melhores competências de pensamento crítico.

No estudo de Ziroldo et al. (2011), podese observar que os alunos das áreas de exatas e humanas, mesmo com estado nutricional adequado apresentaram frequência superior de presença e risco para desenvolvimento da SCN, isso remete ao menor conhecimento do tema em relação aos alunos da área de saúde. Provavelmente o que explica a não relação da síndrome do comer noturno com os estudantes de nutrição e os domínios de escolaridade.

A compulsão alimentar periódica foi outro ponto analisado na população estudada através do questionário de BES. Estudar a compulsão alimentar em universitários é importante e pode ser explicado pela maior tendência dessa população em ter padrões alimentares atípicos, muitas vezes, relacionados a experiências de afetos negativos (MARQUES, 2013). Além disso, é notório que o meio universitário é marcado por muitos desafios, associados ao estresse e ansiedade, refletindo em um padrão alimentar irregular.

Segundo Oliveira et al. (2006), embora a TCAP possa estar presente em homens e mulheres, existe predominância no sexo feminino, na proporção de $3 / 2$, geralmente, tendo início na adolescência.Esse aspecto de maior prevalência no sexo feminino não é evidenciado no estudo, mesmo a amostra sendo composta por o número de mulheres quatro vezes maior do que os homens, o que sugere então uma limitação, não no variável sexo e TCAP, mas no tamanho da amostra, portanto, novos estudos com um número maior de pessoas, possibilitarão uma avaliação mais acurada.

Outro fator que pode levar ao aumento das compulsões alimentares é o estresse, pois durante situações estressantes, o cortisol é liberado para estimular a ingestão de alimentos e o aumento do peso (PIVETTA; GONÇALVES, 2010).

Dessa forma, estudantes de ensino superior cuja maratona diária de estudo é bastante acentuada, podem estar sujeitos a esse tipo de transtorno como demonstra Vitolo et al. (2006), por isso o estudo abordado buscou relacionar a CAP com a escolaridade e o sexo; os resultados obtidos não foram estatisticamente significantes o que possivelmente se explica pelo fato de a amostra não ter avaliado fatores determinantes como a presença da obesidade, sobrepeso e utilização de dados como IMC.

Outro aspecto a ser salientado é a relação entre descontrole alimentar (DA), alimentação emocional (AE) e restrição cognitiva (RC), com questionário TFEQ-R21 que busca revelar comportamentos disfuncionais ligados a hábitos alimentares. Estudos realizados por Pivetta e Gonçalves(2010), em países do hemisfério norte, mostrou que a restrição cognitiva, não se correlacionou 
com os domínios da alimentação emocional e com o descontrole alimentar.

Todavia, por meio dos resultados apresentados na Tabela 3, é possível observar que o domínio de alimentação emocional associou-se fortemente ao descontrole alimentar e seus indicadores, levantando diversas hipóteses: que possivelmente o grupo avaliado neste estudo estivesse sob influência preponderante de fatores desencadeantes da alimentação emocional (por exemplo, estresse, sobrecarga de tarefas e pressões sociais); e também podendo ser decorrente de aspectos tanto da população brasileira, quanto dos próprios hábitos culturais dos indivíduos.

Natacci e Junior (2011), também reforçam essa ideia, tendo em vista que suas análises, em que os escores atribuídos aos três domínios de comportamento mostraram que o TFEQ-R21 ressalta a alimentação emocional, e não a restrição cognitiva, como sendo um dos maiores responsáveis pelo descontrole alimentar e o consumo energético exagerado.

\section{CONCLUSÃO}

Embora não tenhamos encontrado alta prevalência da SCN e da compulsão alimentar em nosso estudo e nem significância, convém salientar que tais transtornos podem estar diretamente associados às consequências das vivências acadêmicas.

$\mathrm{O}$ investimento no estudo dos transtornos alimentares é importante para acriação de uma intervenção adequada, com o intuito de reduzir a incidência tanto da SCN como da compulsão alimentar nos jovens que normalmente está associada às exigências do contexto acadêmico superior.

Por fim, consideramos a contribuição deste estudo para maior aprofundamento das análises efetuadas a respeito da $\mathrm{SCN}$ e ingesta compulsiva diante à realidade dos alunos do ensino superior.

\section{REFERÊNCIAS}

BERNARDI, F. et al. Transtornos alimentares e padrão circadiano alimentar: uma revisão. Rev. psiquiatr., Rio Grande do Sul, v. 209, n. 31, p. 3, 2009.
CALUGI, S et al. Night eating syndrome in class II-III obesity: metabolic and psychopathological features. Int J Obes., v. 33, n. 8, p. 899-904, 2009.

CLEATOR, J.et al. Night eating syndrome: implications for severe obesity. Nutr Diabetes., v. 2, n. 9, 2012.

COLLES, S. L.et al. Night eating syndrome and nocturnal snacking: association with obesity, binge eating and psychological distress. Int J Obes.,v. 31, n. 11, p. 17221730, 2007.

COSTA, M. B. et al. Diferenças na percepção da imagem corporal, no comportamento alimentar e no estado nutricional de universitárias das áreas de saúde e humanas. Rev Psiquiatr Rio Gd Sul.,v. 31, n. 3, p. 192196, 2009.

DUARTE, F. M. et al. Alimentação fora do domicílio de universitários de alguns cursos da área da saúde de uma instituição privada. O Mundo da Saúde., v. 37, n. 3, p. 288-298, 2013.

FISCHER, S. et al. Night eating syndrome in young adults: Delineation from other eating disorders and clinical significance. J Psychiatry Research.,v. 200, n. 2, p. 494501, 2012.

FREITAS, S. et al. Tradução e adaptação para o português da Escala de Compulsão Alimentar Periódica. Rev Bras Psiquiatr.,v. 23, n. 4, p. 215-220, 2001.

GALLANT, A. R. et al. Etiology and Pathophysiology the night-eating syndrome and obesity. Obes Rev., v. 13, n. 6, p. 528-536, 2012.

HARB, A. B. C. et al. Síndrome do comer noturno: aspectos conceituais, epidemiológicos, diagnósticos e terapêuticos. Rev Nutr PUCCAMP., Campinas, v. 23, n.1, p. 127-136, 2010.

HARB, A. B. C. et al. Tradução e adaptação da versão brasileira do Night Eating Questionnaire Translation and adaptation of the Brazilian version of the Night Eating Questionnaire. Cad Saúde Publica., v. 24, n. 6, p. 13681376, 2008. 
MARQUES, S. I. P. Perturbação de ingestão compulsiva, alimentação emocional e síndrome do comer noturno: um estudo comparativo entre sujeitos com peso normal, excesso de peso e obesidade. 2013. 121f. Dissertação (Mestrado em Psicologia clínica) Universidade da Beira Interior, Covilhão, 2013.

MILANO, W. et al. Night eating syndrome: an overview. J Pharm Pharmacol., London, v.64, n.1,p.2-10, 2012.

NATACCI. L. C.; JÚNIOR M. F. The three factor eating questionnaire - R21: translation and administration to Brazilian women. Rev Nutr., v. 24, n. 3, p. 383-394, 2011.

NOLAN, L. J.; GELIEBTER, A. Night eating is associated with emotional and external eating in college students. Eat Behav., v. 13, n. 3, p. 202-206, 2012.

OLIVERA, G. A. et al. A compulsão alimentar na recepção dos profissionais de saúde. Psicologia Hospitalar, v. 4, n. 2, p. 1-18, 2006.

PIVETTA, L. A.; GONÇALVES S. R. Compulsão alimentar e fatores associados em adolescentes de Cuiabá. Cad Saúde Publica., v. 26, n. 2, p. 337-346, 2010.

TAPADINHAS, A. R.; RIBEIRO, J. L. P.Ingestão compulsiva e gestão das emoções na obesidade. Psicologia, Saúde e Doenças., v. 9, supl.1, 2008.

VITOLO, M. R. et al. Prevalência de compulsão alimentar entre universitárias de diferentes áreas de estudo. Rev. psiquiatr., Rio Grande do Sul, v.28, p.20-26, 2006.

ZIROLDO, D. F. et al. Prevalência da síndrome do comer noturno em universitárias. Saúde e Pesqui., v.4,n.3,p.401-406, 2011.

Recebido em:15 de setembro de 2016 Aceito em: 12 abril de 2017 